\title{
Encuesta Nacional sobre la utilización de catéter venoso central en pacientes sometidos a neurocirugía electiva en Chile
}

\author{
Use of central venous catheter in patients undergoing \\ elective neurosurgery in Chile: A national survey
}

Rogers Ortega', Felipe Maldonado², Cristian Rocco³, Paz Ahumada

\begin{abstract}
Objectives: To know, through an online survey, the usual clinical practice of Chilean anesthesiologists regarding central venous catheterization in patients undergoing elective neurosurgery. Material and Methods: An email was sent with a link to a questionnaire to anesthesiologists belonging to the Society of Anesthesiology of Chile (SACH). The questionnaire consisted of an anonymous questionnaire, with multiple-choice questions that included data referring to experience as an anesthesiologist, experience in neuroanesthesia, indication of central venous access in elective neurosurgery, access of choice, technique used, and immediate and late complications. Results: A valid response was received to the online survey by 180 anesthesiologists, which represents a response rate of $50 \%$. Only $14.4 \%$ of the respondents were classified as specialists in neuroanesthesia. The majority acces corresponded to the Internal Jugular. The main indication was the use of vasoactive drugs in $92.18 \%$. Regarding clinical scenarios, $95.4 \%$ of respondents used a central venous route in posterior fossa surgery, while only $9.41 \%$ used it in minimally invasive surgery. $69.3 \%$ of the respondents indicated that they had presented an immediate complication related to the procedure, with arterial puncture being the most frequent with $72.1 \%$. Protocols for perioperative management only existed in $17.8 \%$ of cases. There are no significant differences in the usual clinical practice among specialists in neuroanesthesia and general anesthesiologists in most of the items analyzed.
\end{abstract}

\section{Key words:}

Central venous catheter, neurosurgery, anesthesia

Servicio de Anestesiología y Reanimación, Complejo Asistencial Víctor Ríos Ruiz, Los Ángeles, Chile.

Servicio de Anestesiología y Reanimación, Hospital Clínico Universidad de Chile, Santiago, Chile.

Clínica Las Condes.

Fecha de recepción: 27 de marzo de 2018

Fecha de aprobación: 24 de abril de 2018

ORCID:

https://orcid.org/0000-0002-3353-4776

Correspondencia:

Dr. Rogers Ortega O.

Email: rogerortegao@gmail.com 
Conclusions: From the data obtained, we observed that there is no uniform criterion in the indication, access and control of the central venous catheter in the context of elective neurosurgery. The foregoing could be explained by the scarce protocolization of anesthetic management in these surgeries. It is of interest to verify that there are no differences in clinical practice among anesthesiologists specialized in this area and those unusual in neurosurgical procedures.

\section{RESUMEN}

Objetivos: Conocer por medio de una encuesta online la práctica clínica habitual de los anestesiólogos chilenos respecto a la cateterización venosa central en pacientes sometidos a neurocirugía de carácter electivo. Material y Métodos: Se envió un correo electrónico con un enlace a un cuestionario a anestesiólogos pertenecientes a la Sociedad de Anestesiología de Chile (SACH). La encuesta consistía en un cuestionario de carácter anónimo, con preguntas de selección múltiple que incluía datos referidos a la experiencia como anestesiólogo, experiencia en neuroanestesia, indicación de vía venosa central en neurocirugía electiva, acceso de elección, técnica utilizada y complicaciones inmediatas y tardías. Resultados: Se recibió respuesta válida a la encuesta online por parte de 180 anestesiólogos, lo que representa una tasa de respuesta del 50\%. Sólo el $14,4 \%$ de los encuestados son catalogados como especialistas en neuroanestesia. La vía de acceso de elección corresponde a la Yugular Interna con un 66,8\% de las preferencias. La principal indicación señalada para su utilización fue el uso de drogas vasoactivas en un $92,18 \%$ de los casos. Respecto a escenarios clínicos el 95,4\% de los encuestados utiliza una vía venosa central en cirugía de fosa posterior, mientras que sólo el 9,41\% la utiliza en cirugía mínimanente invasiva. El 69,3\% de los encuestados indica haber presentado alguna vez una complicación inmediata relacionada al procedimiento, siendo la punción arterial la más frecuente con un $72,1 \%$. Protocolos para el manejo perioperatorio sólo existían en $17,8 \%$ de los casos. No existen diferencias significativas en la práctica clínica habitual entre los especialistas en neuroanestesia y anestesiólogos poco habituados en neuroanestesia en la mayoría de los ítems analizados. Conclusiones: A partir de los datos obtenidos por esta encuesta online, observamos que no existe un criterio uniforme en la indicación, vía de acceso y control del catéter venoso central en el contexto de la neurocirugía electiva. Lo anterior, podría explicarse por la escasa protocolización del manejo anestésico en estas cirugías. Es de interés comprobar que no existen grandes diferencias en la práctica clínica entre los anestesiólogos especialistas en esta área y aquellos poco habituales en procedimientos neuroquirúrgicos.

\section{Palabras clave:}

Catéter venoso central, neurocirugía, anestesia

\section{Introducción}

L a instalación de un catéter venoso central (CVC), es un procedimiento ampliamente utilizado en rúrgicas y uúrgicas y en el manejo de pacientes complejos en diversos escenarios clínicos, tanto en los Servicios de Urgencias, Unidades de Pacientes Críticos y Pabellones Quirúrgicos.
En los Estados Unidos se instalan más de 5 millones de CVC por año y alrededor del 15\% de los pacientes presentan alguna complicación derivada de su instalación, las cuales pueden aumentar tanto la estadía hospitalaria como los costos finales para los pacientes o las instituciones. Estas complicaciones pueden ser mecánicas, trombóticas o infecciosas[1],[2]. El tipo de complicaciones se diferencian en función del lugar de inserción, es así como el acceso subcla- 
vio se asocia con bajo riesgo de infección asociado a catéter, pero un mayor riesgo de neumotórax que la cateterización yugular o femoral[2].

Se han descrito intervenciones tendientes a prevenir las complicaciones asociadas a la instalación de CVC. Algunos autores han definido factores dependientes del operador (experiencia, uso de ultrasonido, uso de radioscopía), factores dependientes del paciente y factores dependientes del tipo de catéter (tipo y tamaño), que pueden determinar el riesgo de complicaciones e incidir en su prevención[3],[4].

Los procedimientos neuroquirúrgicos representan un pequeño porcentaje del total de cirugías, pero la naturaleza de estas intervenciones hacen que en muchas ocasiones sea altamente recomendable la utilización de un catéter venoso central y además involucra ciertas particularidades que debemos considerar al momento de escoger un acceso vascular central (retorno venoso, posición del paciente, uso de soluciones hipertónicas, entre otras). Especial relevancia constituye la cirugía en posición sentada, en la cual es imperioso verificar el correcto posicionamiento del catéter venoso central para el manejo de una complicación potencialmente letal como es la embolia aérea venosa[5].

Una encuesta realizada en el Reino Unido el año 2000, entre anestesiólogos miembros de la Sociedad de Neuroanestesia, demostró que no existía un criterio uniforme respecto a la indicación ni vía de acceso para la cateterización venosa central en neuroanestesia electiva[6].

Considerando la variabilidad clínica que se puede observar, es de interés determinar mediante la difusión de un encuesta nacional si existe algún tipo de consenso entre los anestesiólogos chilenos respecto a la indicación, vía de acceso, técnica utilizada y complicaciones observadas en pacientes sometidos a cirugía craneal de carácter electivo.

\section{Objetivo}

Conocer la práctica clínica habitual de los anestesiólogos chilenos en el uso de catéter venoso central en el manejo de los pacientes adultos sometidos a craneotomía de carácter electivo.

\section{Objetivos específicos:}

- Describir vía de acceso de elección.

- Describir principales indicaciones para su instalación.

- Describir probable diferencia en el enfrentamiento entre anestesiólogos con experiencia en proce- dimientos neuroquirúrgicos y anestesiólogos poco habituales en estas cirugías.

- Describir complicaciones inmediatas y a mediano plazo relacionadas al procedimiento.

\section{Materiales y Métodos}

Encuesta diseñada en forma conjunta por médicos anestesiólogos, especialmente dedicados al área neuroquirúrgica del Complejo Asistencial Víctor Ríos Ruiz, Los Ángeles y Hospital Clínico Universidad de Chile, Santiago. Encuesta anónima, de selección múltiple, que incluye datos relacionados a la experiencia profesional en anestesia y en neuroanestesia, indicación, vía de acceso y complicaciones asociadas a la cateterización venosa central para procedimientos neuroquirúrgicos electivos. La encuesta fue difundida y contestada en formato electrónico, entre el 01 de marzo y 15 de abril de 2017. Información y promoción de la encuesta se realizó en la página web Sociedad Anestesiología de Chile (https://www.sachile.cl/). Se enviaron correos electrónicos dirigido a anestesiólogos miembros de la Sociedad. El correo entregó acceso a un enlace con una encuesta online, diseñada utilizando el software tipo SurveyMonkey. ${ }^{\circledR}$ (https:// es.surveymonkey.com/r/R6K6228)

El cuestionario consistió en 16 preguntas, la mayor parte de tipo test con respuesta única o múltiple, algunas de ellas con opción a realizar comentarios. No se realizó consentimiento informado ya que la propia participación en la encuesta se consideró consentimiento implícito.

La primera parte del cuestionario estuvo dirigida a conocer la experiencia como anestesiólogo y en neuroanestesia. La segunda parte tiene relación con conocer la indicación, vía de acceso y control de la posición de catéter venoso central en neurocirugía electiva. La última parte del cuestionario contenían preguntas sobre eventuales complicaciones asociadas a este tipo de procedimiento. La encuesta detallada está disponible en el Anexo 1.

Para efectos de esta encuesta se definió como especialista en neuroanestesia a quienes trabajan de forma frecuente en anestesia neuroquirúrgica, en centros donde se realizan más de 10 neurocirugías por semana.

Se realizó un análisis estadístico descriptivo de la población a encuestar, incluyendo representación gráfica de los resultados. El marco de referencia corresponde a los anestesiólogos miembros de la Sociedad de Anestesiología de Chile incluidos en la base de datos de la Sociedad. Los resultados se presentaron 
como números, frecuencias y porcentajes de cada respuesta. Las variables cualitativas se compararon utilizando Test exacto de Fisher o Chi-cuadrado, según corresponde. Se consideró un valor de $p<0,05$ como significativo. El análisis estadístico de los datos se realizó con el programa estadístico IBM® SPSS ${ }^{\circledR}$, versión 22.

Este estudio consideró las recomendaciones CHERRIES para reportes de encuestas online[7].

\section{Resultados}

La encuesta fue recibida correctamente por 360 anestesistas, de los cuales se recibieron 201 respuestas. Se eliminaron 21 encuestas por respuestas incompletas (sólo 1 ó 2 preguntas contestadas) por lo cual se analizaron 180 encuestas, lo que representa una tasa de respuesta del $50 \%$.

De acuerdo a la definición de especialista en neuroanestesia propuesta, el $14,4 \%$ de los participantes corresponden a especialistas en anestesia neuroquirúrgica (Tabla 1). En relación a la experiencia como anestesiólogo el $57,4 \%$ de los encuestados posee más de 10 años de experiencia. Sólo el 17,8\% de los encuestados indicó que en su lugar de trabajo existía un protocolo de manejo perioperatorio de pacientes sometidos a neurocirugía electiva.

La vía de acceso para el CVC se observa en la Figura 1. Destaca la similitud de resultados entre los especialistas y no especialistas, representando el abordaje yugular interno el más frecuentemente utilizado $(66,8 \%)$.

La indicación de CVC en las distintas cirugías se muestra en la Figura 2. La cirugía de fosa posterior se

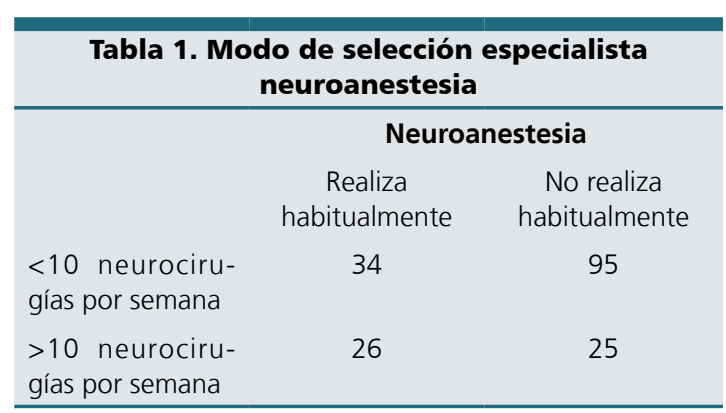

Anestesiólogos habituales en neurocirugía en centros con más de 10 neurocirugías a la semana.

consideró indicación de cateterización venosa central en el 95,4\% de los casos, mientras que en la cirugía mínimamente invasiva en sólo el 9,41\%. No existe diferencia significativa entre ambos grupos.

En relación a la técnica utilizada el $58,8 \%$ de los encuestados señala que siempre utiliza acceso ecoguiado y un $7,2 \%$ nunca lo utiliza.

Respecto a la verificación de la posición final de la punta del catéter, sólo el 62,9\% lo considera como una práctica fundamental en neuroanestesia. El modo de verificar la posición del catéter se encuentra en la Tabla 2.

Referido a las complicaciones asociadas al procedimiento, un $69,3 \%$ de los encuestados señala haber presentado en alguna oportunidad una complicación inmediata, siendo la punción arterial las más frecuentemente señalada. Los médicos especialistas en neuroanestesia refieren presentar más complicaciones inmediatas que los médicos no especialistas, en particular el fracaso de inserción del catéter con una diferencia significativas entre ambos grupos $(p<$

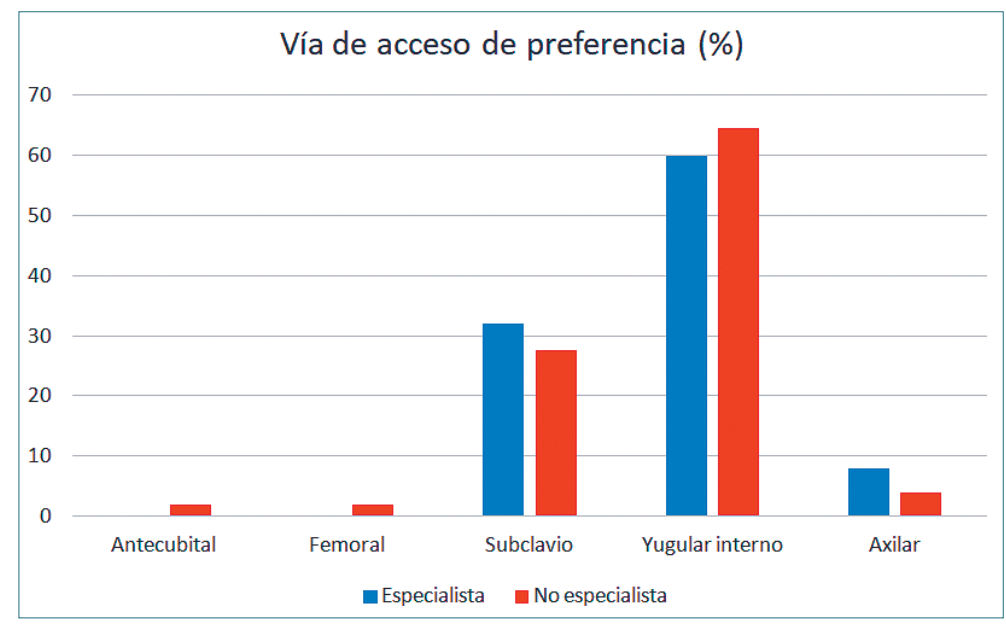

Figura 1. Acceso central de elección. Los resultados se muestran como porcentaje del total de respuestas de cada grupo (especialistas en neuroanestesia y no especialistas). 


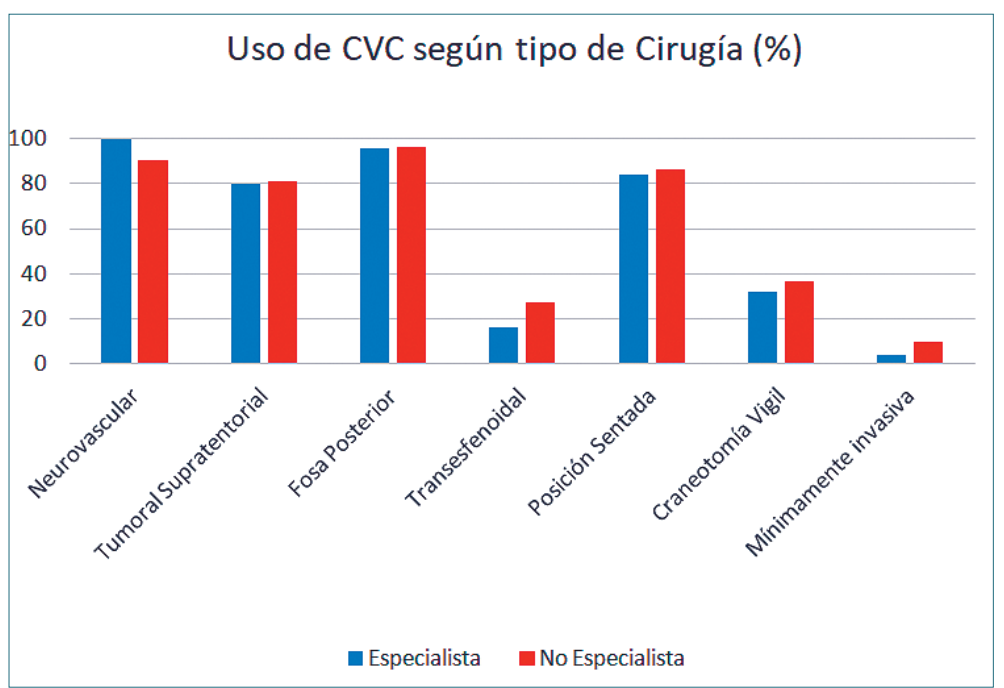

Figura 2. Indicación de CVC en diferentes neurocirugías. Los resultados se muestran como porcentaje del total de respuestas de cada grupo (especialistas en neuroanestesia y no especialistas).

Tabla 2. Manera de verificación posición final punta del catéter

\begin{tabular}{lcc}
\hline Forma de verificar posición punta catéter & Especialista (\%) & No especialista (\%) \\
ECG continuo & 16 & 8,6 \\
Ecografía & 16 & 9,3 \\
Radioscopía & 44 & 58,9 \\
Otro & 8 & 7,9 \\
No verifica & 16 & 15,2
\end{tabular}

Los resultados se muestran como porcentaje del total de respuestas de cada grupo (especialistas en neuroanestesia y no especialistas).

0,05) (Tabla 3). Más de la mitad de los encuestados señaló desconocer la existencia de complicaciones a mediano plazo $(55,9 \%)$.

El resultado de todas las preguntas de la encuesta se detalla en la Tabla 4.

\section{Discusión}

La cateterización venosa central es un procedimiento ampliamente utilizado en pacientes quirúrgicos de mediana a alta complejidad y representa una práctica habitual en el contexto de la neurocirugía. La seguridad y confianza de este acceso es un aspecto importante en el cuidado de estos pacientes. El objetivo de esta encuesta nacional fue conocer las prácticas clínicas habituales de los anestesiólogos chilenos respecto a la utilización de vía venosa central en neurocirugía de carácter electivo.
Es de interés comprobar que no existe un consenso en la indicación de utilización de vía venosa central en los diferentes escenarios clínicos propuestos. Particular atención supone la no indicación de cateterización venosa central en neurocirugía en posición sentada $(14,8 \%)$, a pesar del riesgo incrementado que supone esta posición de presentar una complicación potencialmente letal como lo es la embolia aérea venosa[8]. De igual manera sólo el $64,2 \%$ de los anestesistas consideran la aspiración de aire en caso de embolia aérea como criterio para utilizar una vía venosa central y el 62,7\% considera fundamental verificar posición final de la punta del catéter, a pesar que la correcta localización del catéter (hasta 2 cm distal a la unión de vena cava superior y aurícula derecha) aumenta la efectividad de la aspiración de aire en caso de embolia aérea[5]. La radioscopía fue la forma más frecuente de verificar la posición final del catéter, concordante con las recomendaciones de la 
Tabla 3. Complicación inmediata alguna vez observada

\begin{tabular}{lcc}
\hline Complicación inmediata secundaria a CVC & & No especialista (\%) \\
Complicación inmediata & Especialista (\%) & 66,4 \\
Neumotórax & $87,5^{*}$ & 19,1 \\
Punción arterial & 36 & 53,9 \\
Mal posición & 69 & 36,8 \\
Arritmias & 28 & 17,1 \\
Fracaso inserción & 24 & 32,9 \\
\hline
\end{tabular}

(Los resultados se presentan como porcentaje del total de respuestas para cada grupo).

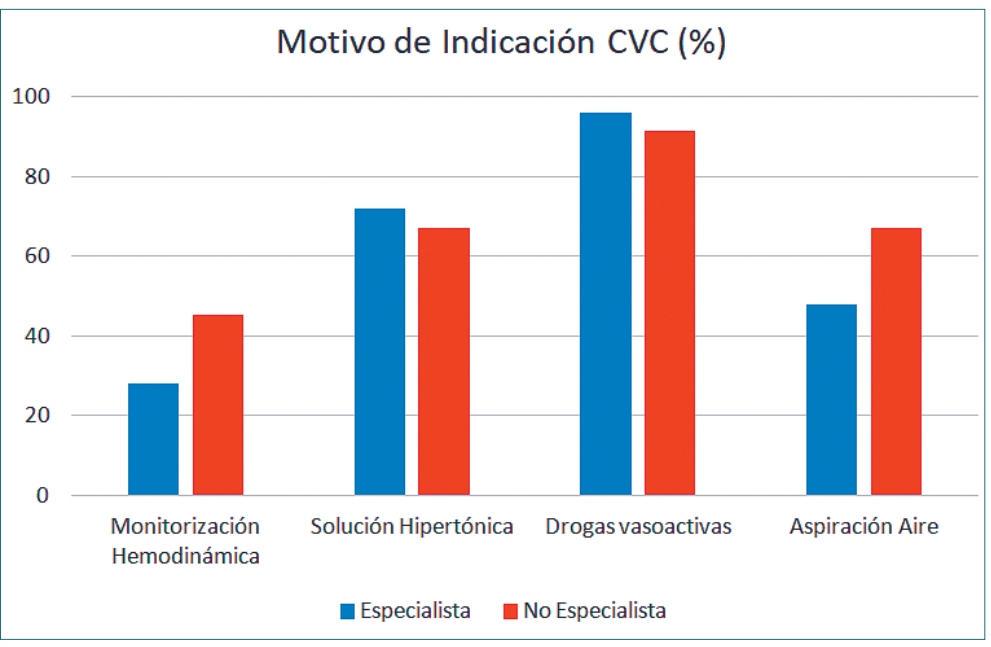

Figura 3. Principal indicación de CVC en neurocirugía electiva. Los resultados se muestran como porcentaje del total de respuestas de cada grupo (especialistas en neuroanestesia y no especialistas). literatura que incluyen también la electrocardiografía continua y la radiografía de tórax como métodos de confirmación de la posición de la punta del catéter[9]. Con respecto a lo último, no se presentaron diferencias significativas entre el grupo de especialistas en neuroanestesia y los no especialistas.

La principal razón para indicar cateterización venosa central en este tipo de pacientes fue la administración de drogas vasoactivas (92,1\%). En este punto sería interesante determinar qué porcentaje de neurocirugías efectivamente requieren apoyo con drogas vasoactivas, y que en nuestra opinión corresponde a un porcentaje menor de los casos, a excepción de los cuadros de hemorragia subaracnoidea (HSA) que pueden precipitar graves complicaciones cardiovasculares[10].

Consideramos que esta variabilidad clínica observada puede guardar relación con la escasa protocolización del manejo anestésico de estos pacientes que de acuerdo a datos de esta encuesta alcanza sólo el

\section{$17,8 \%$.}

En la mayoría de los casos el acceso de elección es el yugular interno (66,8\%), seguido por el abordaje subclavio (29,7\%). Un 58,8\% de los encuestados señala siempre utilizar ultrasonido, práctica avalada por la evidencia que demuestra una significativa mejora en efectividad y seguridad del acceso vascular guiado por ultrasonido comparado con el abordaje basado en reparos anatómicos, en particular el acceso yugular interno[11],[12]. Además, a pesar que se ha sugerido que la cateterización de la vena yugular interna pudiera impedir el adecuado retorno venoso, situación trascendente en pacientes con hipertensión endocraneana, diferentes estudios han concluido que este efecto no es significativo[13],[14]. El acceso femoral y el acceso periférico vía antecubital sólo representan el acceso de elección en el 1,71\% de los casos respectivamente. Cabe señalar que la recomendación para la elección del sitio de inserción del catéter debe estar basado en el juicio clínico, experiencia y habilidad del 


\section{Tabla 4. Resumen Resultados Encuesta}

\begin{tabular}{|c|c|c|c|}
\hline 1. Años de experiencia como anestesiólogo & $\begin{array}{l}<5 \text { años } \\
5-10 \text { años } \\
<10 \text { años }\end{array}$ & $\begin{array}{l}N 180 \\
35(21,1 \%) \\
35(21,1 \%) \\
110(57,5 \%)\end{array}$ & \\
\hline 2. Habitual en neuroanestesia & $\begin{array}{l}\text { Sí } \\
\text { No } \\
\text { Ocasionalmente }\end{array}$ & $\begin{array}{l}N 180 \\
61(33,8 \%) \\
32(17,7 \%) \\
87(48,3 \%)\end{array}$ & \\
\hline 3. Neurocirugías electivas en su centro semanalmente & $\begin{array}{l}<5 \\
5-10 \\
>10\end{array}$ & $\begin{array}{l}\text { N } 178 \\
70(39,3 \%) \\
57(32,0 \%) \\
51(26,6 \%)\end{array}$ & \\
\hline 4. Se considera especialista en neuroanestesia & $\begin{array}{l}\text { Sí } \\
\text { No }\end{array}$ & $\begin{array}{l}N 180 \\
28(15,5 \%) \\
152(84,4 \%)\end{array}$ & \\
\hline 5. Protocolo manejo perioperatorio & $\begin{array}{l}\text { Sí } \\
\text { No }\end{array}$ & $\begin{array}{l}\text { N } 179 \\
32(17,8 \%) \\
147(82,1 \%)\end{array}$ & \\
\hline $\begin{array}{l}\text { 6. Instalación CVC según tipo de Cirugía } \\
\text { Tipo de cirugía } \\
\text { Neurovascular } \\
\text { Tumoral supratentorial } \\
\text { Fosa posterior } \\
\text { Transesfenoidal hipófisis } \\
\text { Posición sentada } \\
\text { Craneotomía vigil } \\
\text { Mínimamente invasiva }\end{array}$ & $\begin{array}{l}N \\
174 \\
174 \\
175 \\
174 \\
175 \\
165 \\
170\end{array}$ & $\begin{array}{l}\text { Sí } \\
160(91,1 \%) \\
35(20,1 \%) \\
167(95,4 \%) \\
46(26,4 \%) \\
150(85,7 \%) \\
59(35,7 \%) \\
16(9,4 \%)\end{array}$ & $\begin{array}{l}\text { No } \\
14(8,09) \\
140(80,4 \%) \\
8(4,5 \%) \\
129(74,4 \%) \\
26(14,8 \%) \\
106(64,2 \%) \\
155(91,1 \%)\end{array}$ \\
\hline 7. Principal razón indicación CVC & $\begin{array}{l}\text { Monitoreo hemodinámico } \\
\text { Soluciones hipertónicas } \\
\text { Drogas vasoactivas } \\
\text { Aspiración de aire } \\
\text { Otras }\end{array}$ & $\begin{array}{l}\text { N } 179 \\
76(42,4 \%) \\
121(67,6 \%) \\
165(92,1 \%) \\
115(64,2 \%) \\
13(7,2 \%)\end{array}$ & \\
\hline 8. Acceso de elección & $\begin{array}{l}\text { Yugular interno } \\
\text { Subclavio } \\
\text { Femoral } \\
\text { Ante cubital }\end{array}$ & $\begin{array}{l}\text { N } 175 \\
117(66,8 \%) \\
52(29,7 \%) \\
3(1,71 \%) \\
3(1,71 \%)\end{array}$ & \\
\hline 9. Acceso ecoguiado & $\begin{array}{l}\text { Siempre } \\
\text { Ocasionalmente } \\
\text { Rara vez } \\
\text { Nunca }\end{array}$ & $\begin{array}{l}\text { N } 180 \\
106(58,8 \%) \\
47(27,6 \%) \\
14(7,78 \%) \\
13(7,2 \%)\end{array}$ & \\
\hline 10. Verificación punta de catéter & $\begin{array}{l}\text { Sí } \\
\text { No }\end{array}$ & $\begin{array}{l}N 178 \\
112(62,9 \%) \\
66(37,0 \%)\end{array}$ & \\
\hline 11. Forma verificación punta catéter & $\begin{array}{l}\text { ECG } \\
\text { Radioscopia } \\
\text { Ecografía } \\
\text { No considero importante } \\
\text { Otras }\end{array}$ & $\begin{array}{l}N 179 \\
18(10,0 \%) \\
101(56,4 \%) \\
19(10,6 \%) \\
27(15,0 \%) \\
14(7,8 \%)\end{array}$ & \\
\hline
\end{tabular}




\begin{tabular}{|c|c|c|}
\hline 12. Control radiografía tórax & $\begin{array}{l}\text { Sí } \\
\text { No }\end{array}$ & $\begin{array}{l}N 180 \\
136(75,5 \%) \\
44(24,4 \%)\end{array}$ \\
\hline 13. Complicación Inmediata & $\begin{array}{l}\text { Sí } \\
\text { No }\end{array}$ & $\begin{array}{l}\text { N } 176 \\
122(69,3 \%) \\
54(30,6 \%)\end{array}$ \\
\hline 14. Tipo de Ccomplicación inmediata & $\begin{array}{l}\text { Neumotórax } \\
\text { Punción arterial } \\
\text { Mal posición catéter } \\
\text { Arritmias } \\
\text { Fracaso inserción } \\
\text { Otras }\end{array}$ & $\begin{array}{l}\text { N } 140 \\
40(28,5 \%) \\
101(72,1 \%) \\
63(45 \%) \\
32(22,8 \%) \\
65(46,4 \%) \\
4(2,8 \%)\end{array}$ \\
\hline 15. Complicaciones a mediano plazo & $\begin{array}{l}\text { Bacteriemia y/o sepsis } \\
\text { Trombosis } \\
\text { No } \\
\text { No sabe }\end{array}$ & $\begin{array}{l}\text { N } 177 \\
14(7,9 \%) \\
15(8,4 \%) \\
49(27,6 \%) \\
99(55,9 \%)\end{array}$ \\
\hline 16. Registro complicaciones & $\begin{array}{l}\text { Sí } \\
\text { No } \\
\text { No sabe }\end{array}$ & $\begin{array}{l}\text { N } 180 \\
67(37,2 \%) \\
64(35,5 \%) \\
49(27,2 \%)\end{array}$ \\
\hline
\end{tabular}

(N: número de respuestas; (\%): porcentaje de respuesta del número de encuestas válidas contestadas).

operador[9]. La escasa utilización del acceso femoral es concordante con la literatura, en la cual se recomienda evitar este acceso en pacientes adultos para la prevención de infecciones intravasculares asociadas a catéter[15]. Respecto al acceso central vía periférico, consideramos que es una alternativa válida, que se debe tener en consideración, en particular en este tipo de cirugías, en las cuales el catéter es retirado en general al corto plazo (48 - 72 horas), por lo cual las complicaciones trombóticas[16], frecuentemente relacionadas a este acceso, no tendrían incidencia relevante: Cabe señalar que este tipo de acceso es utilizado en neurocirugía electiva en varios centros a nivel mundial y puede constituir un acceso ideal en el intra y posoperatorio inmediato de estos pacientes[6] Consideramos que su casi nula utilización por parte de los anestesiólogos chilenos se debe en gran medida a ausencia del insumo, poca familiaridad en su uso y gran experiencia y destreza con los otros accesos. Importante recalcar que no existe evidencia clara que demuestre la superioridad del catéter de inserción periférica (en particular PICC) sobre el CVC en el contexto del paciente quirúrgico y se requieren de más estudios para documentar sus ventajas[17].

Es interesante describir que el 69,3\% de los anestesiólogos señala haber presentado alguna complicación inmediata relacionada a la instalación de CVC, destacando la punción arterial y el fracaso en la in- serción del catéter en el lugar escogido inicialmente, como las dos situaciones que más frecuentemente se presentan. Si bien es cierto que las publicaciones existentes demuestran una considerable variación en el rango de complicaciones desde el 1 - 26\%[1], consideramos que la cifra excesivamente alta descrita en esta encuesta no es comparable con el porcentaje mencionado anteriormente, debido fundamentalmente a diferencias metodológicas, entre las que destaca el desconocimiento del número total CVC insertados por parte de los anestesiólogos encuestados, apartado que no fue considerado dentro de los objetivos de este estudio. La diferencia significativa observada entre ambos grupos se puede explicar porque probablemente el grupo de especialistas tiene un número mayor de procedimientos que el grupo catalogado como no especialista en neuroanestesia.

Más de la mitad de los encuestados $(55,9 \%)$ señala ignorar ocurrencia de alguna complicación a mediano plazo derivado de la instalación y manejo de un CVC, lo que nos puede indicar el escaso seguimiento que tenemos de estos pacientes, mas allá del intra y posoperatorio inmediato.

Indudablemente, este tipo de encuesta presenta ciertas limitaciones, entre ellas la tasa de respuesta, que en nuestro caso alcanzó el 50\%, que si bien es cierto representa un buen número de respuestas, es bastante inferior a una encuesta similar realizada en el 
Reino Unido[6], por lo que cabe la posibilidad que la muestra no sea representativa de todos los anestesiólogos de nuestro país. Esta tasa de respuesta se puede explicar por la falla en la recepción de los correos electrónicos y también porque el tema presentado no era de interés general. Otra limitación es la definición operativa utilizada en esta encuesta para la clasificación como especialista en neuroanestesia, lo anterior debido al desconocimiento de alguna forma de estandarización y registro utilizado en nuestro país en este sentido. El número de neuroanestesistas definidos por nuestra clasificación $(n=26)$ es muy similar al resultado de la pregunta que incluye la consideración personal como criterio para catalogarse como especialista $(n=28)$. De todas maneras estimamos que este número representa una cifra muy menor en relación a anestesiólogos con formación y entrenamiento específico en esta área.

En resumen, creemos que los datos recogidos por esta encuesta nos permiten describir la actuación clínica habitual de los anestesiólogos chilenos frente a la cateterización venosa central en pacientes sometidos a neurocirugía electiva. Esperamos que este trabajo sea el punto de partida para objetivar datos que permitan establecer recomendaciones para disminuir la variabilidad clínica en el manejo de estos pacientes.

Agradecimientos: Se agradece a la Sociedad Chilena de Anestesiología por actividades de información y promoción, y en particular a los anestesiólogos chilenos por su buena disposición a responder esta encuesta. El autor agradece a la Dra. Neus Fàbregas y Dr. Ricard Valero por su colaboración y recomendaciones durante todo el desarrollo de este trabajo.

\section{Referencias}

1. Lathey RK, Jackson RE, Bodenham A, Harper D, Patle V. A multicentre snapshot study of the incidence of serious procedural complications secondary to central venous catheterisation. Anaesthesia [Internet]. Wiley; 2016 Dec 16;72(3):328-34.

2. Parienti JJ, Mongardon N, Megarbane B. Intravascular Complications of Central Venous Catheterization by Insertion Site. Journal of Vascular Surgery [Internet]. Elsevier BV; 2016 Mar;63(3):846.

3. Domino KB, Bowdle TA, Posner $\mathrm{KL}$, Spitellie PH, Lee LA, Cheney FW. Injuries and Liability Related to Central Vascular Catheters. Anesthesiology [Internet]. Ovid Technologies (Wolters Kluwer Health); 2004 Jun;100(6):14118.

4. Hove LD, Steinmetz J, Christoffersen JK, M? ?ller A, Nielsen J, Schmidt $\mathrm{H}$. Analysis of Deaths Related to Anesthesia in the Period 1996-2004 from Closed Claims Registered by the Danish Patient Insurance Association.
Anesthesiology [Internet]. Ovid Technologies (Wolters Kluwer Health); 2007 Apr;106(4):67580.

5. Iturri Clavero F, Honorato C, Ingelmo Ingelmo I, Fàbregas Julià N, Rama-Maceiras P, Valero R, et al. Consideraciones preoperatorias y manejo neuroanestesiológico intraoperatorio. Revista Española de Anestesiología y Reanimación [Internet]. Elsevier BV; 2012 Nov; 59:3-24.

6. Mills SJ, Tomlinson AA. The use of central venous cannulae in neuroanaesthesia. Anaesthesia [Internet]. Wiley; 2001 May 2;56(5):465-70

7. Eysenbach $\mathrm{G}$. Improving the Quality of Web Surveys: The Checklist for Reporting Results of Internet E-Surveys (CHERRIES). Journal of Medical Internet Research [Internet]. JMIR Publications Inc.; 2004 Sep 29;6(3):e34.

8. Mirski MA, Lele AV, Fitzsimmons L, Toung TJK. Diagnosis and Treatment of Vascular Air Embolism. Anesthesiology [Internet]. Ovid Technologies (Wolters Kluwer Health); 2007 Jan;106(1):164-77.
9. \&NA; Practice Guidelines for Central Venous Access. Anesthesiology [Internet]. Ovid Technologies (Wolters Kluwer Health); 2012 Mar;116(3):539-73.

10. McLaughlin N, Bojanowski M, Denault A. Early myocardial dysfunction following subarachnoid haemorrhage. British Journal of Neurosurgery [Internet]. Informa UK Limited; 2005 Apr;19(2):1417.

11. Hayashi $H$, Amano M. Does ultrasound imaging before puncture facilitate internal jugular vein cannulation? Prospective randomized comparison with landmark-guided puncture in ventilated patients. Journal of Cardiothoracic and Vascular Anesthesia [Internet]. Elsevier BV; 2002 Oct; 16(5):572-5.

12. Dietrich $C F$, Horn $R$, Morf $S$, Chiorean L, Dong Y, Cui X-W, et al. Ultrasound-guided central vascular interventions, comments on the European Federation of Societies for Ultrasound in Medicine and Biology guidelines on interventional ultrasound. Journal of Thoracic Disease [Internet]. AME Publishing Company; 2016 


\section{Sep;8(9):E851-E868.}

13. Woda RP, Miner ME, McCandless C, McSweeney TD. The effect of right internal jugular vein cannulation on intracranial pressure. J Neurosurg Anesthesiol. 1996 Oct;8(4):286-92.

14. Vailati D, Lamperti M, Subert $M$, Sommariva A. An ultrasound study of cerebral venous drainage after internal jugular vein catheterization. Crit Care Res Pract. 2012;2012:1-5.
15. O'Grady NP, Alexander M, Burns LA, Dellinger EP, Garland J, Heard SO, et al. Summary of Recommendations: Guidelines for the Prevention of Intravascular Catheter-related Infections. Clinical Infectious Diseases [Internet]. Oxford University Press (OUP); 2011 May 1;52(9):1087-99.

16. Fletcher JJ, Wilson TJ, Rajajee $\mathrm{V}$, Stetler WR, Jacobs TL, Sheehan KM, et al. A Randomized Trial of Central Venous Catheter Type and Thrombosis in Critically III Neurologic Patients. Neurocritical Care [Internet]. Springer Nature; 2016 Feb 2;25(1):20-8.

17. Turcotte $S$, Dubé $S$, Beauchamp G. Peripherally Inserted Central Venous Catheters Are Not Superior to Central Venous Catheters in the Acute Care of Surgical Patients on the Ward. World Journal of Surgery [Internet]. Springer Nature; 2006 Jul 21;30(8):1605-19. 